This is an Open Access article, distributed under the terms of the Creative Commons Attribution licence (http://creativecommons.org/licenses/by/4.0/), which permits unrestricted re-use, distribution, and reproduction in any medium, provided the original work is properly cited.

\title{
Turbulence Heating ObserveR - satellite mission proposal
}
A. Vaivads ${ }^{1} \uparrow$, A. Retinò ${ }^{2}$, J. Soucek ${ }^{3}$, Yu. V. Khotyaintsev ${ }^{1}$, F. Valentini ${ }^{4}$, C. P. Escoubet ${ }^{5}$, O. Alexandrova ${ }^{6}$, M. André ${ }^{1}$, S. D. Bale ${ }^{7,8}$, M. Balikhin ${ }^{9}$, D. Burgess ${ }^{10}$, E. Camporeale ${ }^{11}$, D. Caprioli ${ }^{12}$, C. H. K. Chen ${ }^{13}$, E. Clacey ${ }^{14}$, C. M. Cully ${ }^{15}$, J. De Keyser ${ }^{16}$, J. P. Eastwood ${ }^{13}$, A. N. Fazakerley ${ }^{17}$, S. Eriksson ${ }^{18}$, M. L. Goldstein ${ }^{19}$, D. B. Graham ${ }^{1}$, S. Haaland ${ }^{20,21}$, M. Hoshino ${ }^{22}$, H. Ji ${ }^{23}$, H. Karimabadi ${ }^{24}$, H. Kucharek ${ }^{25}$, B. Lavraud ${ }^{26,27}$, F. Marcucci $^{28}$, W. H. Matthaeus ${ }^{29}$, T. E. Moore ${ }^{19}$, R. Nakamura ${ }^{30}$, Y. Narita $^{30}$, Z. Nemecek ${ }^{31}$, C. Norgren ${ }^{1}$, H. Opgenoorth ${ }^{1}$, M. Palmroth ${ }^{32}$, D. Perrone ${ }^{33}$, J.-L. Pinçon ${ }^{34}$, P. Rathsman ${ }^{14}$, H. Rothkaehl ${ }^{35}$, F. Sahraoui ${ }^{2}$, S. Servidio ${ }^{4}$, L. Sorriso-Valvo ${ }^{36}$, R. Vainio ${ }^{37}$, Z. Vörös ${ }^{30}$ and R. F. Wimmer-Schweingruber ${ }^{38}$

\footnotetext{
${ }^{1}$ Swedish Institute of Space Physics, Uppsala, 75121, Sweden
}

${ }^{2}$ Laboratoire de Physique des Plasmas, Ecole Polytechnique, Palaiseau, F-91128, France

${ }^{3}$ Institute of Atmospheric Physics, Czech Academy of Sciences, Praha, CZ-14131, Czech Republic

${ }^{4}$ Dipartimento di Fisica, Università della Calabria, Rende (CS), I-87036, Italy

${ }^{5}$ ESTEC, European Space Agency, 2200 AG Noordwijk, Netherlands

${ }^{6}$ LESIA, Observatoire de Paris, 92190 Meudon, France

${ }^{7}$ Physics Department, University of California, Berkeley, CA 94720, USA

${ }^{8}$ Space Sciences Laboratory, University of California, Berkeley, CA 94720, USA

${ }^{9}$ Department of Automatic Control and Systems Engineering, University of Sheffield, Sheffield, UK

${ }^{10}$ School of Physics and Astronomy, Queen Mary University of London, London E1 4NS, UK

${ }^{11}$ Center for Mathematics and Computer Science (CWI), 1090 GB Amsterdam, Netherlands

${ }^{12}$ Department of Astrophysical Sciences, Princeton University, Ivy Lane, Princeton, NJ 08540, USA

${ }^{13}$ Department of Physics, Imperial College London, London SW7 2AZ, UK

${ }^{14}$ OHB Sweden, 16440 Kista, Sweden

${ }^{15}$ Department of Physics and Astronomy, University of Calgary, Calgary, Alberta, Canada

${ }^{16}$ Space Physics Division, Belgian Institute for Space Aeronomy, Brussels, Belgium

${ }^{17}$ Mullard Space Science Laboratory, University College London, Holmbury St. Mary, Dorking, Surrey, RH5 6NT, UK

${ }^{18}$ Laboratory for Atmospheric and Space Physics, University of Colorado, Boulder, CO, USA

${ }^{19}$ NASA Goddard Space Flight Center, Greenbelt, Maryland, MD 20771, USA

${ }^{20}$ Max-Planck-Institut für Sonnensystemforschung, 37077 Göttingen, Germany

${ }^{21}$ Department of Physics and Technology, University of Bergen, Norway

${ }^{22}$ Department of Earth and Planetary Science, University of Tokyo, Tokyo, Japan

$\dagger$ Email address for correspondence: andris.vaivads@gmail.com 
${ }^{23}$ Department of Astrophysical Sciences and Princeton Plasma Physics Laboratory, Princeton University, Princeton, NJ 08544, USA

${ }^{24}$ SciberQuest, Del Mar, CA 92014, USA

${ }^{25}$ Institute for the Study of Earth, Oceans, and Space, University of New Hampshire, NH 03824-3525, USA

${ }^{26}$ Institut de Recherche en Astrophysique et Planétologie, Université de Toulouse, France

${ }^{27}$ Centre National de la Recherche Scientifique, UMR 5277, Toulouse, France

${ }^{28}$ INAF/IAPS, Rome, Italy

${ }^{29}$ Department of Physics and Astronomy, Bartol Research Institute, University of Delaware, Newark, DE 19716, USA

${ }^{30}$ Space Research Institute, Austrian Academy of Sciences, Graz, Austria

${ }^{31}$ Faculty of Mathematics and Physics, Charles University, Prague 18000, Czech Republic

${ }^{32}$ Earth Observation Unit, Finnish Meteorological Institute, Helsinki, Finland

${ }^{33}$ European Space Agency, Science and Robotic Exploration Directorate, ESAC, Madrid, Spain

${ }^{34}$ LPC2E/CNRS, Laboratoire de Physique et Chimie de l'Environnement et de l'Espace, Orléans, France

${ }^{35}$ Space Research Centre, Polish Academy of Sciences, Warsaw, Poland

${ }^{36}$ Nanotec/CNR, U.O.S. LICRYL di Cosenza, Ponte P. Bucci, Cubo 31C, I-87036 Rende, CS, Italy

${ }^{37}$ Department of Physics and Astronomy, University of Turku, Turku, FI-20014, Finland

${ }^{38}$ Institute of Experimental and Applied Physics, Christian-Albrechts-University, Kiel, Germany

(Received 18 July 2016; revised 24 August 2016; accepted 25 August 2016)

The Universe is permeated by hot, turbulent, magnetized plasmas. Turbulent plasma is a major constituent of active galactic nuclei, supernova remnants, the intergalactic and interstellar medium, the solar corona, the solar wind and the Earth's magnetosphere, just to mention a few examples. Energy dissipation of turbulent fluctuations plays a key role in plasma heating and energization, yet we still do not understand the underlying physical mechanisms involved. THOR is a mission designed to answer the questions of how turbulent plasma is heated and particles accelerated, how the dissipated energy is partitioned and how dissipation operates in different regimes of turbulence. THOR is a single-spacecraft mission with an orbit tuned to maximize data return from regions in near-Earth space - magnetosheath, shock, foreshock and pristine solar wind - featuring different kinds of turbulence. Here we summarize the THOR proposal submitted on 15 January 2015 to the 'Call for a Medium-size mission opportunity in ESAs Science Programme for a launch in 2025 (M4)'. THOR has been selected by European Space Agency (ESA) for the study phase.

Key words: plasma heating, plasma properties, space plasma physics

\section{Scientific objectives}

Ordinary matter in the Universe is primarily in the plasma state. It is the hot dilute plasma between galaxies and galaxy clusters, and not stars, that dominates baryonic matter. Hot dilute plasma can also be found within galaxies, such as in the interstellar medium, in outer atmospheres and stellar winds of stars, coronas or accretion disks. Astrophysical plasmas are turbulent, and dissipation of turbulent fluctuations due to large-scale shear motions, shock waves, jets and other large-scale 
instabilities and processes leads to continuous plasma heating and to acceleration of plasma particles. Understanding basic processes of particle energization in turbulent magnetized plasmas is of fundamental importance to understand the evolution of the Universe. While turbulent fluctuations in astrophysical plasmas reach scales as large as entire galaxies, most of the irreversible dissipation of energy associated with turbulent fluctuations occurs at the very small scales - the kinetic scales. The efficiency of plasma heating, the partitioning of energy transferred to different particle species, the acceleration of particles to high energies - all are strongly governed by kinetic processes that determine how turbulent electromagnetic fluctuations dissipate. Thus, plasma processes at kinetic scales will directly affect the large-scale properties of astrophysical plasmas.

Turbulence Heating ObserveR (THOR) will be the first mission flown in space dedicated to plasma turbulence and heating. It will explore the kinetic processes that determine plasma heating and particle energization, their efficiency for different plasma species and their relative importance in different turbulent regimes, see table 1. THOR will provide closure of these fundamental questions by making detailed in situ measurements of the closest available dilute and turbulent magnetized plasmas at unprecedented temporal and spatial resolution. THOR focuses on key science regions - pristine solar wind, Earth's bow shock and interplanetary shocks and compressed solar wind regions downstream of shocks, see figure 1. These regions are selected because of their differing turbulent fluctuation characteristics, and they reflect similar astrophysical environments. In addition, both spatial and temporal characteristic plasma scales in the key science regions are sufficiently large, so that the particle instruments are able to resolve the kinetic scales, see figure 2. THOR will spend long intervals also in other regions, such as the magnetotail, where a lot of important science questions will be addressed. However, the design of payload and mission is optimized to maximize the scientific return from the key science regions. The THOR spacecraft will carry, for the first time, a comprehensive payload tailored to explore plasma energization in turbulence, with both fields and particle instrumentation that will allow the simultaneous resolution of both the turbulent fluctuations and the signature of the resultant plasma energization. The payload consists of mature instruments with recent flight heritage. THOR will also open new paths by providing measurements that go beyond our current theoretical expectations, thus allowing the exploration of new physics and challenging our current theories.

THOR science directly addresses the Cosmic Vision question 'How does the Solar System work?' by studying basic processes occurring 'From the Sun to the edge of the Solar System'. By quantifying the fundamental processes involved, the advances made by the THOR mission will extend beyond the Solar System to plasmas elsewhere in the Universe. THOR will provide understanding of fundamental plasma processes with applications to very different astrophysical, solar system and laboratory plasma environments. Due to studies involving a variety of space missions, including Cluster, THEMIS and MMS (and in the near future, missions such as Solar Orbiter and Solar Probe Plus) we now understand many aspects of plasma turbulence, such as its three-dimensional structure, owing to multi-spacecraft observations. However, how the turbulence dissipates and heats the surrounding medium and energizes particles is not at all well understood. That is the unique mission of THOR.

Here we summarize the THOR proposal, submitted on 15 January 2015 to the 'Call for a Medium-size mission opportunity in ESAs Science Programme for a launch in 2025 (M4)'. On 4 June 2015, THOR was selected by ESA for a detailed study phase.

THOR will provide experimental support to answer three major science questions, which are given in table 1 and briefly summarized below. THOR will also allow 


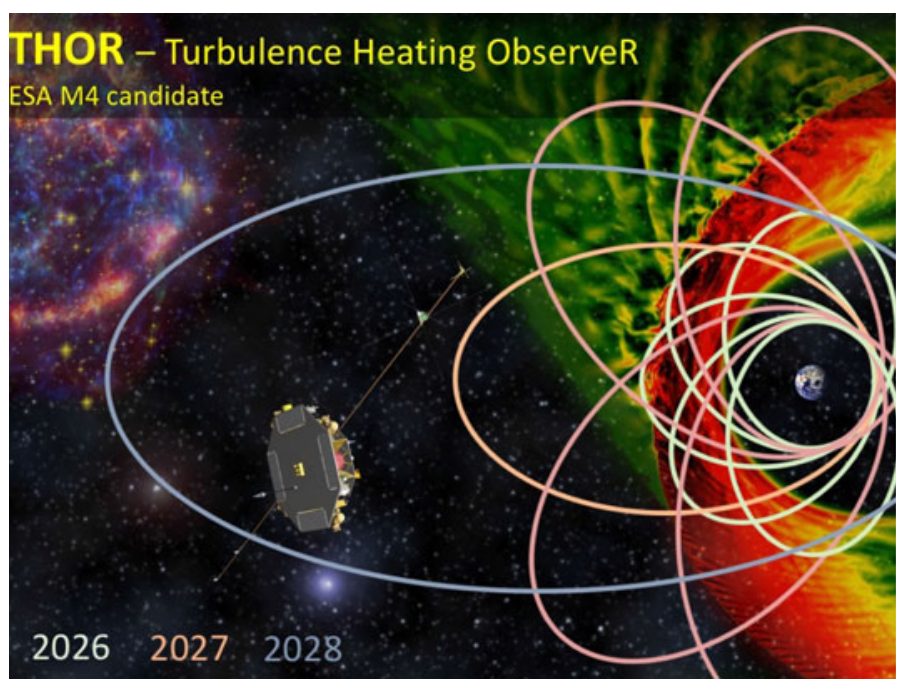

FIGURE 1. Schematic drawing of the THOR spacecraft and its orbits during the 3 years of the nominal science mission. During the first year the focus is on the bow shock and magnetosheath, during the second and third years the focus is on the pristine solar wind and foreshock. Courtesy: NASA, OHB-Sweden, FMI and Vlasiator (von Alfthan et al. 2014).

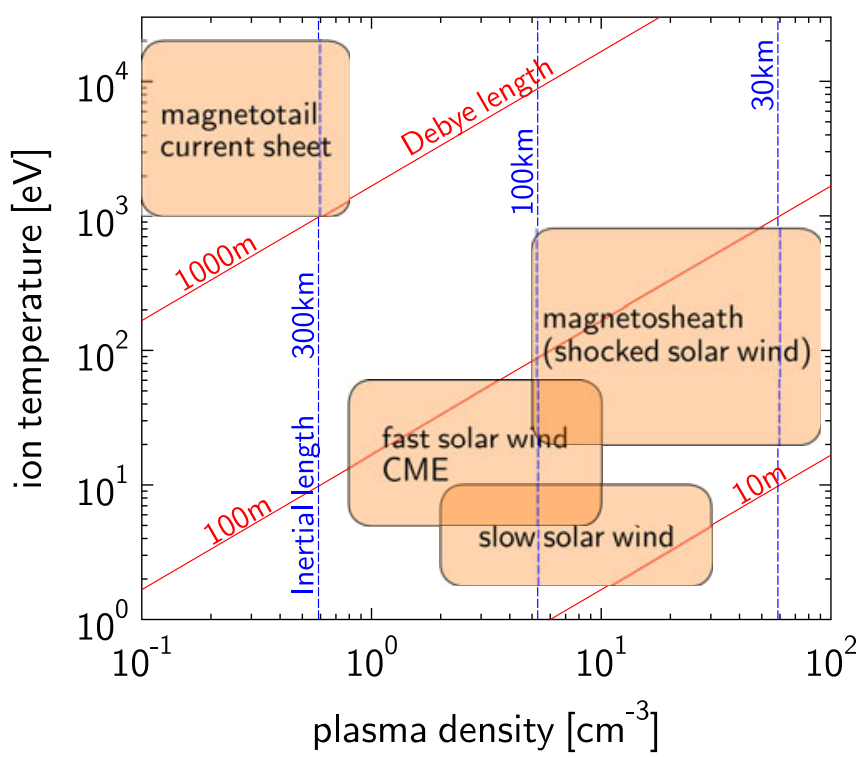

FIGURE 2. Typical plasma parameter ranges and characteristic spatial scales, Debye length and ion inertial length, in the different regions encountered by THOR.

studies of additional science questions, which are not detailed here: turbulence at fluid scales, reconnection in the solar wind, reconnection generated turbulence, electromagnetic emission generation in solar wind such as Type II and Type III bursts, magnetospheric physics like plasma jet front kinetic structure, space weather science and many others. 
Science theme: Turbulent energy dissipation and particle energization

1. How is plasma heated and particles accelerated?

2. How is the dissipated energy partitioned?

3. How does dissipation operate in different regimes of turbulence?
Science questions

Wave mode identification and spectra; effects of wave damping on plasma; coherent structure identification; effects of coherent structures on plasma

Among electrons, protons and heavier ions; between heating and particle acceleration

Turbulence for different values of typical plasma parameters, e.g. amplitude of turbulent fluctuations, plasma $\beta$, plasma composition, homogeneity, Mach number.

TABLE 1. The overall science theme and science questions of the THOR mission.

Science question I: How is plasma heated and particles accelerated? Most of the turbulent energy dissipation occurring in collisionless plasmas is expected to occur at kinetic scales. A variety of fluctuations, different types of linear/nonlinear waves and coherent structures, see example in figure 3, can operate at kinetic scales and are associated with different heating and acceleration mechanisms as indicated by numerical simulations (Servidio et al. 2009; Chandran et al. 2010; Camporeale \& Burgess 2011; Servidio et al. 2012; Karimabadi et al. 2013; TenBarge \& Howes 2013; Caprioli \& Spitkovsky 2014; Valentini et al. 2014). Yet only limited in situ observations of dissipation and associated energization at kinetic scales are available in near-Earth turbulent plasmas (Retinò et al. 2007; Sundkvist et al. 2007; Sahraoui et al. 2009; Perri et al. 2012; šafránková et al. 2013; Chen et al. 2014), due to the lack of dedicated measurements. Consequently, the exact contribution of the different mechanisms to the overall energy dissipation is basically unexplored from the observational point of view due to the insufficient resolution of temporal, angular and energy measurements of particle distribution functions. Different turbulent dissipation mechanisms result in different modifications of the particle distribution functions, e.g. formation of anisotropies, beams, fine-scale structure and high-energy tails, which can be localized in space at kinetic scales (Camporeale \& Burgess 2011; Haynes, Burgess \& Camporeale 2014). Therefore, in addition to the electromagnetic turbulence, it is crucial to measure distribution functions with both high temporal resolution and high velocity-space resolution, to understand how turbulent heating and particle acceleration operates, see figure 4.

Science question II: How is the dissipated energy partitioned? The energy dissipated at kinetic scales is partitioned between the different particle species and also between thermal heating and energetic particle acceleration. Determining the physics underlying this partitioning through in situ measurements is crucial for understanding how plasma energization works. It can also help interpreting the energization mechanisms behind the electromagnetic radiation measured from distant astrophysical objects during key phenomena. Many signatures exist in the turbulent solar wind and shock regions indicating that plasma is continuously being energized. As an example, solar wind observations of ion temperature over many astronomical units are not consistent with an adiabatic expansion (Richardson et al. 1995; Borovsky \& Gary 2014) indicating 

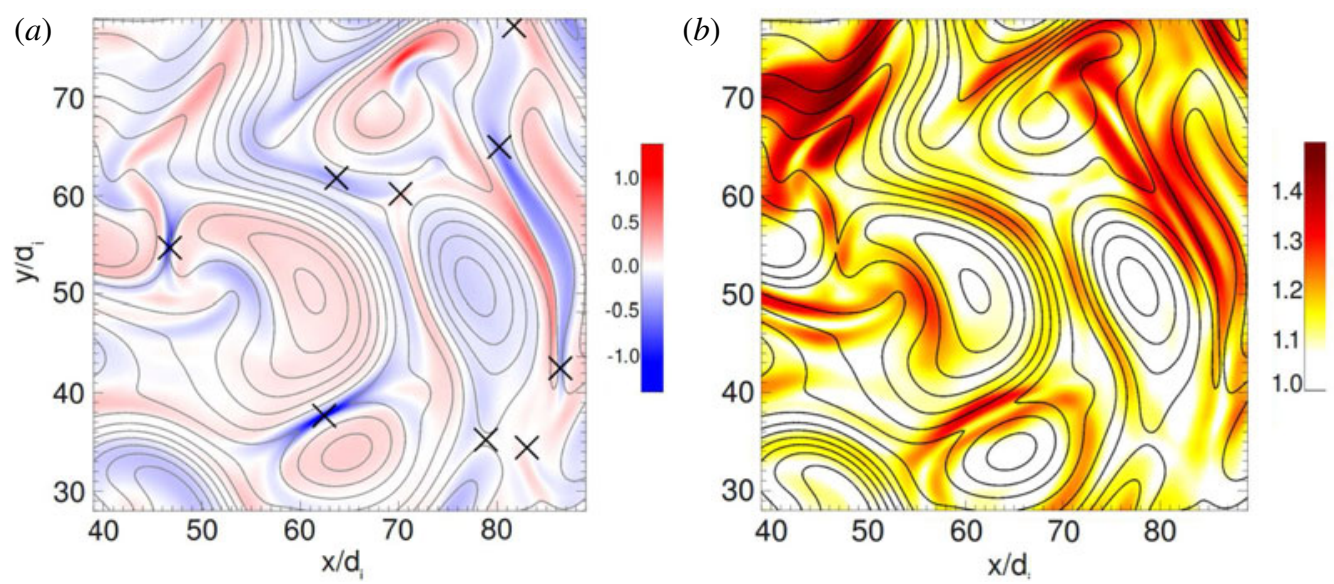

FIgURE 3. Turbulent ion heating in two-dimensional hybrid Vlasov-Maxwell simulations. (a) Current density (colour) and magnetic field lines, possible reconnection sites are indicated by crosses. (b) The proton temperature anisotropy (colour). The kinetic deformations of the particle velocity distributions are concentrated around coherent structures that are located near the peaks of current (Servidio et al. 2012).

that solar wind plasma is being continuously heated. Yet, solar wind electrons and protons show different temperatures, e.g. electrons are typically cooler than protons in the fast wind while hotter in the slow wind (Marsch 2006) suggesting that different heating mechanisms are at work for electrons and ions. Furthermore, heavier ions (alpha particles in particular) seem to be preferentially heated with respect to protons, the temperature ratio being more than mass proportional (Kasper, Lazarus \& Gary 2008), see also figure 5. Non-Maxwellian features of distribution functions such as beams and energetic tails are also found both in the solar wind and in planetary, interplanetary and termination shock regions (Burgess et al. 2005; Eastwood et al. 2005; Lucek et al. 2005; Marsch 2006; Liu et al. 2007), indicating that both heating and acceleration are at work. Most of these signatures have been provided by large-scale observations of turbulent fluctuations and particle distribution functions, while major turbulent dissipation is expected at kinetic scales. Yet high-resolution coordinated field and particle measurements in the solar wind and shock regions resolving kinetic scales are scarce. The THOR mission will provide such measurements and allow important questions about energy partition in turbulent dissipation to be answered.

Science question III: How does dissipation operate in different regimes of turbulence? This question addresses which turbulent fluctuations and dissipation mechanisms are dominant under different plasma conditions and how plasma energization works in the variety of plasmas present in the solar system and beyond in the wider universe. The near-Earth space provides an excellent laboratory to test this, thanks to the different regions sampled by THOR along its orbit, see figure 1. These regions are characterized by different values of typical plasma parameters such as amplitude of turbulent fluctuations, plasma $\beta$, plasma composition, homogeneity, collisionality, Mach number, system size, etc. Key regions are the pristine fast and slow solar wind, interaction regions between flows like the fast and slow solar wind, shocks and associated sheath regions. Such near-Earth regions are representative of a number of 
(a)

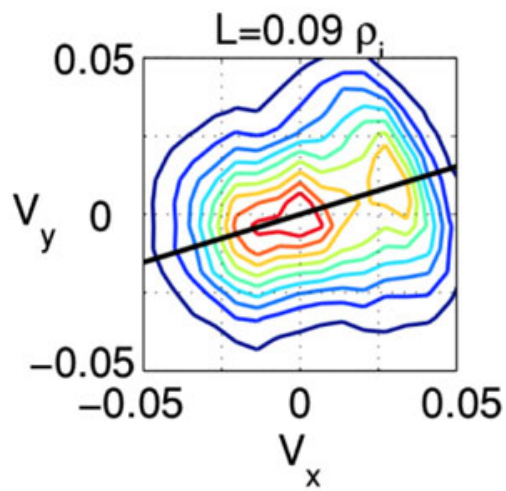

(c)

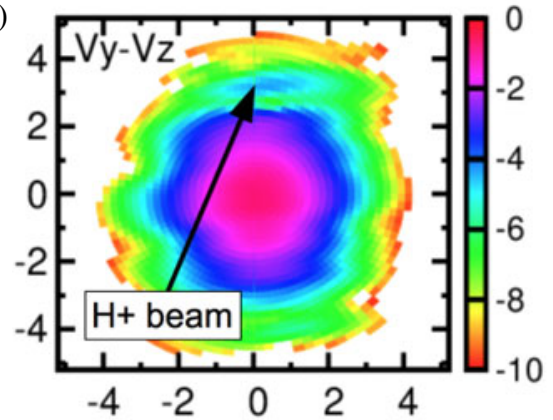

(b)

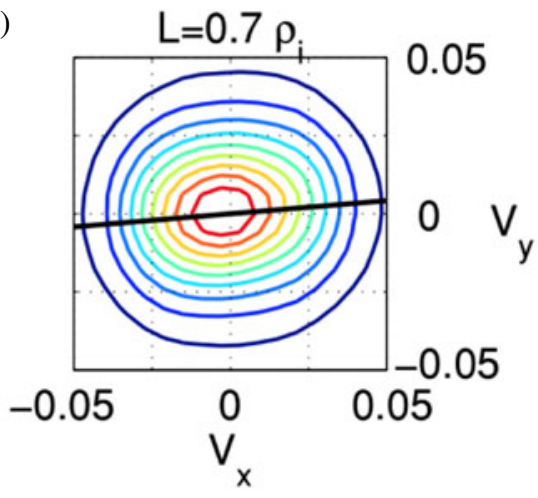

(d)

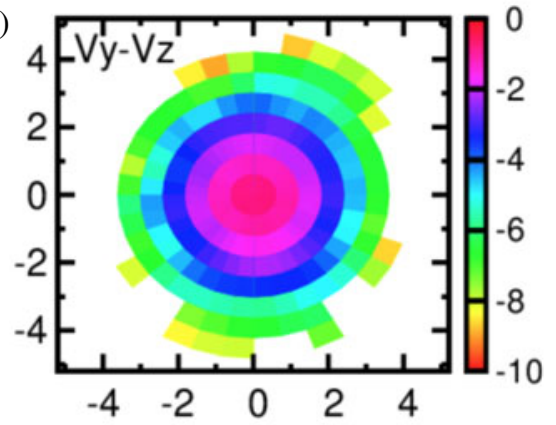

FIGURE 4. Illustration of the need for high temporal, spatial and energy resolution of particle instruments based on turbulent plasma simulations. (a,b) Electron distribution function for high $(a)$ and low $(b)$ spatial resolution (Camporeale \& Burgess 2011). To resolve electron beams requires resolution of the distribution function at electron kinetic scales. $(c, d)$ Ion distribution function for high $(c)$ and low $(d)$ velocity space resolution. To resolve ion beams requires high velocity space (energy and angular) resolution.

astrophysical turbulent environments, so that the identification of dominant dissipation mechanisms by THOR will help understand heating in distant astrophysical objects where in situ measurements are not available.

\section{Mission}

THOR is designed as a Sun-pointing spinning satellite with a spin period of $\sim 30 \mathrm{~s}$, see figure 6. The spacecraft design heritage is drawn from the Freja satellite (Lundin, Haerendel \& Grahn 1994). Spin is necessary to deploy long wire antennas to achieve accurate and sensitive electric field measurements, as well as for a complete angular coverage by energetic particle detectors and to enable accurate magnetic field calibration. A lower spin rate decreases the time resolution for full energetic particle characterization, and higher spin rates degrade the angular resolution of velocity distribution measurements and have an impact on the fuel budget. The solar cells for power production are mounted on dedicated panels at the edges of the top platform and can radiate from their back side. In addition, the lower platform has a smaller diameter than the top platform, further improving the radiative cooling to space. Side panels are not used, which reduces mass and allows more cooling possibilities to 

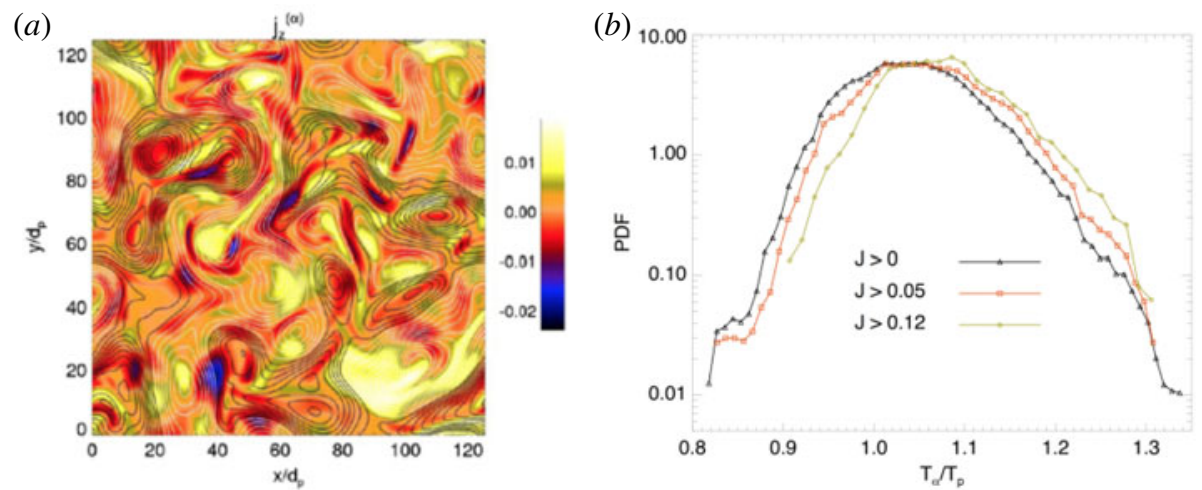

FIgURE 5. Heating of ions with different mass. (a) Contour plots (shaded colours) of the out-of-plane alpha particle current density. Magnetic field lines are indicated by black/white lines. (b) Distribution of the alpha-to-proton temperature anisotropy (right) for three different thresholds of the total current (proton + alphas) (Perrone et al. 2013, 2014).
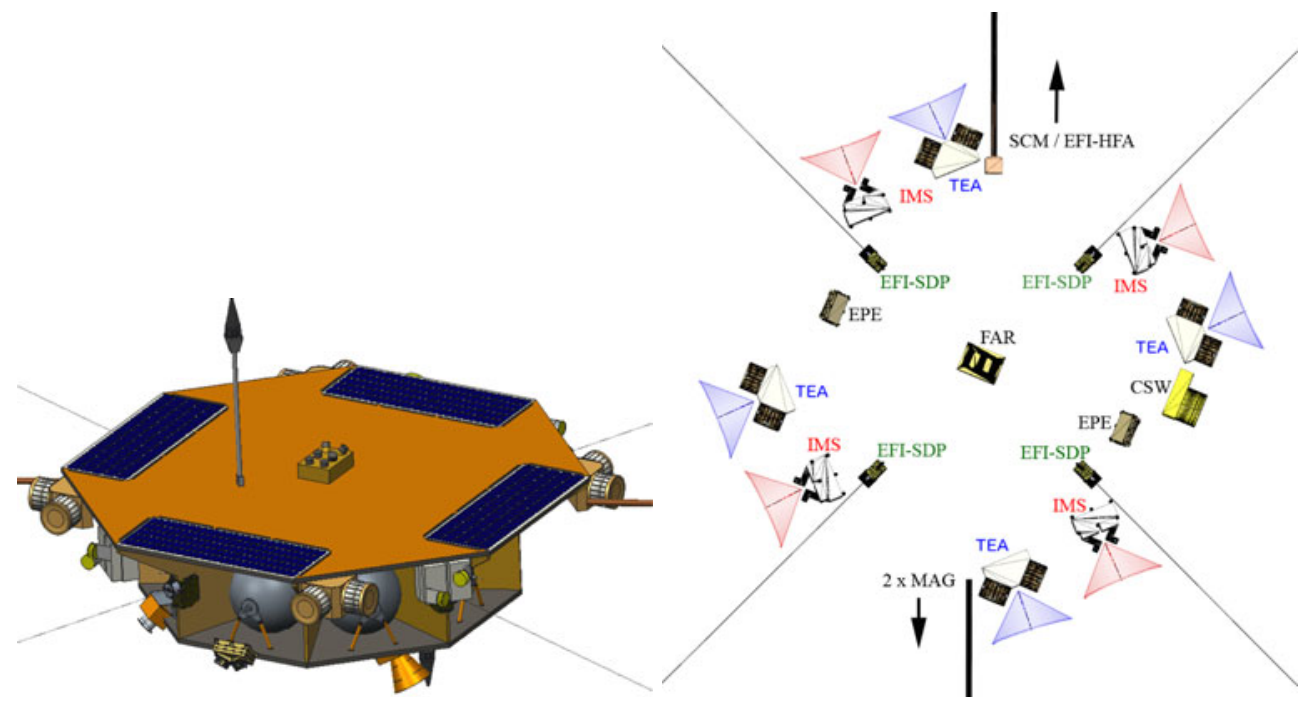

FIGURE 6. THOR, three-dimensional view and instrument accommodation. The field of view of the instruments is marked with shaded triangles. Wire booms extend up to $50 \mathrm{~m}$ away from the spacecraft. The solid booms are $6 \mathrm{~m}$ long.

internally mounted equipment. The sides, however, are covered with single-layer foils with electrically conductive outer surfaces. Vertical shear walls connect the top and bottom platforms and provide mounting surfaces for the payload and system units.

The THOR orbit has been designed to have three phases, as summarized in table 2 . Figure 7 shows the expected position of magnetopause and bow shock. In addition, the orbital coverage of THOR is coloured in grey. At launch, THOR will be injected into a geocentric $4 R_{E} \times 16 R_{E}$ orbit with the orbital plane close to the ecliptic plane. During the first year (Phase 1), the low perigee allows the telemetry to be maximised, and the apogee being slightly outside the nominal bow shock location allows an optimization 


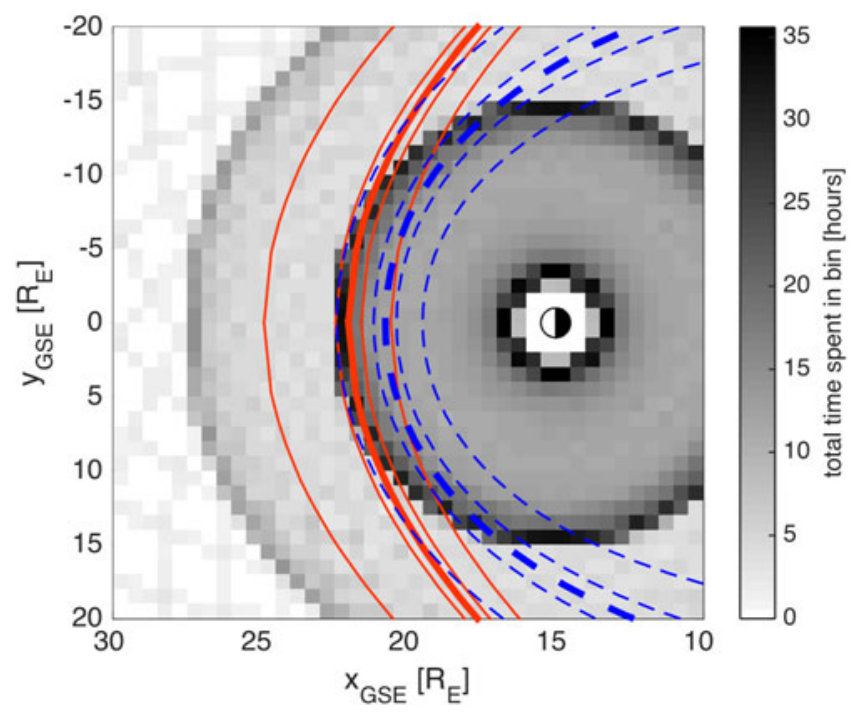

FIGURE 7. The nominal location of the bow shock and magnetopause for expected solar wind conditions 2025/26 based on the daily averaged OMNI data and common models. The thick red-solid and blue-dashed lines show the mean positions of the bow shock and magnetopause respectively, while the thinner lines show the minimum, $25 \%$ percentile, $75 \%$ percentile and maximum locations. The grey scale shows the orbital coverage of THOR.

Phase

(1) 1 st year

(2) 2nd year

(3) 3rd year
Orbit

$4 \times 16 R_{E}$

$4 \times 26 R_{E}$ $14 \times 60 R_{E}$
Regions of main science focus

Bow shock and magnetosheath

Solar wind and foreshock

Pristine solar wind and interplanetary shocks

TABLE 2. THOR mission phases during the nominal science mission. Orbit parameters can be adjusted based on the expected solar cycle development.

of the time spent at the bow shock. During the second year (Phase 2), the orbit is designed with a $26 R_{E}$ apogee in order to spend longer time periods in the undisturbed solar wind and foreshock. Finally, in Phase 3, THOR is put in the approximate orbit $14 \times 60 R_{E}$ where both long time periods in pristine solar wind during apogee and long periods around bow shock at perigee are possible. The final orbit shall be stable, yet have the possibility to use a lunar gravity assist to change the orbit during the extended mission, for example to go to the L1 Lagrangian point.

THOR will be equipped with an Active Spacecraft Potential (ASP) controller, which reduces the positive spacecraft potential by emitting indium ions. This allows for much more accurate plasma measurements at low energies comparable to the spacecraft potential and provides a better satellite potential environment improving the electric field measurements. On THOR, ASP is located on the shadow side of the spacecraft and emits the ion beam away from the spacecraft in the anti-Sunward direction.

THOR is a highly focused scientific mission with payload and systems slimmed to target the main science goals. However, several variations of the above scenario 


$\begin{array}{lccc}\text { Instrument } & \text { Measured quantity } & \text { Range } & \text { Max. cadence } \\ \text { MAG } & \text { Magnetic field } & \text { DC-64 Hz } & 128 \mathrm{sps} \\ \text { SCM } & \text { Magnetic field } & 1 \mathrm{~Hz}-200 \mathrm{kHz} & 524 \mathrm{ksps} \\ \text { EFI } & \text { Electric field } & \text { DC-200 kHz } & 524 \mathrm{ksps} \\ \text { FAR } & \text { Fast ion moments } & & 32 \mathrm{sps} \\ \text { FWP } & \text { Fields and waves } & & 524 \mathrm{ksps} \\ \text { TEA } & \text { Electron distribution } & 1 \mathrm{eV}-20 \mathrm{keV} & 5 \mathrm{~ms} \\ \text { IMS } & \text { Ion distribution } & 5 \mathrm{eV}-32 \mathrm{keV} & 150 \mathrm{~ms} \\ \text { CSW } & \text { Cold solar wind ions } & 20 \mathrm{eV}-200 \mathrm{keV} & 150 \mathrm{~ms} \\ \text { EPE } & \text { Energetic particles } & \mathrm{e}^{-}: 20 \mathrm{keV}-700 \mathrm{keV} & 7.5 \mathrm{~s} \\ & & \mathrm{i}^{+}: 20 \mathrm{keV}-8 \mathrm{MeV} & \\ \text { PPU } & \text { Particle data products } & & \end{array}$

TABLE 3. THOR payload. The numbers will be further updated during the study phase.

not jeopardizing these goals are easy to foresee. Depending on available launch opportunities, the inclination and perigee altitude may be changed. During an extended mission THOR would have enough propulsion to be moved to L1 and act as a solar wind monitor. The orbit can be adjusted to increase the synergy with other missions.

\section{Payload}

The THOR payload is summarized in tables 3 and 4. The baseline payload includes DC and AC magnetometers (MAG, SCM), four wire probe antennas and three orthogonal dipole antennas to measure DC and AC electric fields in three dimensions (EFI). All measurements from these electromagnetic field sensors will be processed by dedicated electronics modules contained within the FWP instrument box. The advanced particle instrumentation is designed to enable very high time-resolution measurements of particle distributions. The Faraday cup instrument (FAR) measures the ion density, temperature and flow velocity. Ion and electron three-dimensional particle distribution functions are measured using electrostatic analysers (TEA, CSW and IMS). The IMS instrument will allow the different ion species to be differentiated. The TEA electron analyser will sample the thermal electron distribution at a very high cadence and suprathermal electrons at a lower rate. A dedicated instrument, CSW, will be included to provide high-resolution optimized measurements of the drifting cold solar wind ions. Higher energy electrons and ions are monitored with a solid state detector (EPE) at a lower time resolution. Data measured by the IMS, TEA, CSW and EPE analysers will be processed by a common digital processor unit (PPU) to reconstruct the particle distribution functions and compute moments. The baseline payload consists almost entirely of broadly tested technology, with heritage from recent missions (e.g. Cluster, STEREO, RBSP, MMS) combined with newly developed concepts already selected in the context of future missions (e.g. BepiColombo, Solar Orbiter, Solar Probe Plus). Most of the units and building blocks of the payload have recent flight heritage and their performance, constraints and resource utilization are well characterized.

There are many technical advances involving the THOR payload and the THOR mission. Some of them are: increased sensitivity of electric and magnetic field measurements, significantly increased accuracy of electric field measurements and increased temporal and velocity space resolution of particle distribution functions. 
Instrument

$\begin{array}{lc}\text { MAG } & \text { Fluxgate magnetometer } \\ \text { SCM } & \text { Search coil magnetometer } \\ \text { EFI } & 2 \times \text { double probes, } 3 \times \text { boom } \\ \text { FAR } & \text { Faraday cup } \\ \text { FWP } & \text { EM field and wave receiver } \\ \text { TEA } & \text { Electron spectrometer } \\ \text { IMS } & \text { Ion spectrometer with TOF } \\ \text { CSW } & \text { Electrostatic analyser } \\ \text { EPE } & \text { Solid state detector } \\ \text { PPU } & \text { Digital electronics }\end{array}$

Harnesses

Total (including margin)
Mass (kg) Power (W) TRL

Heritage

$\begin{array}{ccr}1 & 3 & 8 \\ 2.4 & 1 & 5 \\ 13.2 & 3 & 5^{\mathrm{a}} \\ 4.8 & 4.8 & 7 \\ 7.2 & 19 & 5 \\ 32.4 & 30 & 5 \\ 28.8 & 34 & 6 \\ 8 & 10 & 6 \\ 4.8 & 5 & 6 \\ 7.2 & 25 & 5 \\ 20 & & \\ 133.8 & 134.8 & \end{array}$
Bepi, VEX, MMS, SolOrb
Cluster, MMS
Cluster, RBSP, JUICE
Spectr-R
SolOrb, JUICE
STEREO, SolOrb, MMS
Cluster, MMS, STEREO
SolOrb
SolOrb
SolOrb

TABLE 4. THOR payload. Solid booms are not included in the instrument mass totals, although harness mass has been included for instruments on those booms (MAG, EFI and SCM). The numbers will be further updated during the study phase. Acronyms: Technical Readiness Level (TRL), BepiColombo (Bepi), Solar Orbiter (SolOrb), JUpiter ICy moons Explorer (JUICE), Venus Express (VEX), Solar TErrestrial RElations Observatory (STEREO), Van Allen Probes (RBSP), Magnetospheric Multiscale (MMS), Electromagnetic (EM), time-of-flight unit (TOF).

${ }^{a}$ EFI-SDP has TRL 7, EFI-HFA has TRL 5.

For the first time mass resolved ions are observed down to kinetic scales. As one example, in figure 8 we show how the Sun-pointing orientation of the spacecraft will allow the satellite potential to be used to measure density gradients with very high accuracy. This has not been possible with earlier satellites having their spin axes at large angles with respect to the Sunward direction. For other advantages of Sun-pointing spacecraft see also Vaivads et al. (2011).

The THOR spacecraft will be designed with strict constraints on electromagnetic cleanliness taken into consideration (Soucek et al. 2016). This is required to ensure that the sensitive electromagnetic measurements performed by the THOR payload are not strongly perturbed by interference from the spacecraft platform and from other instruments. These requirements, while stringent, are readily achievable, based on experience from previous missions such as Cluster and MMS.

The payload operations concept is based on simultaneous and coordinated operation of all instruments. The baseline is to operate the payload continuously, however some of the particle instruments may be switched off during parts of the orbit outside the key science regions in order to extend their life time. The payload will generate two science data streams transferred to the spacecraft mass memory:

(i) Survey data covering the full time at low time resolution. The full Survey data are downloaded to ground.

(ii) Burst data covering the full time in the Regions of Interest (ROI) at high time resolution. Burst data also contain several minutes of high-resolution waveform (HRWF) data. The telemetry rate is not sufficient to download the full volume of Burst data on each orbit, and therefore selective data downlink will be used for the Burst data, where the Burst intervals for downlink will be selected based on the science priorities using the Survey data. 


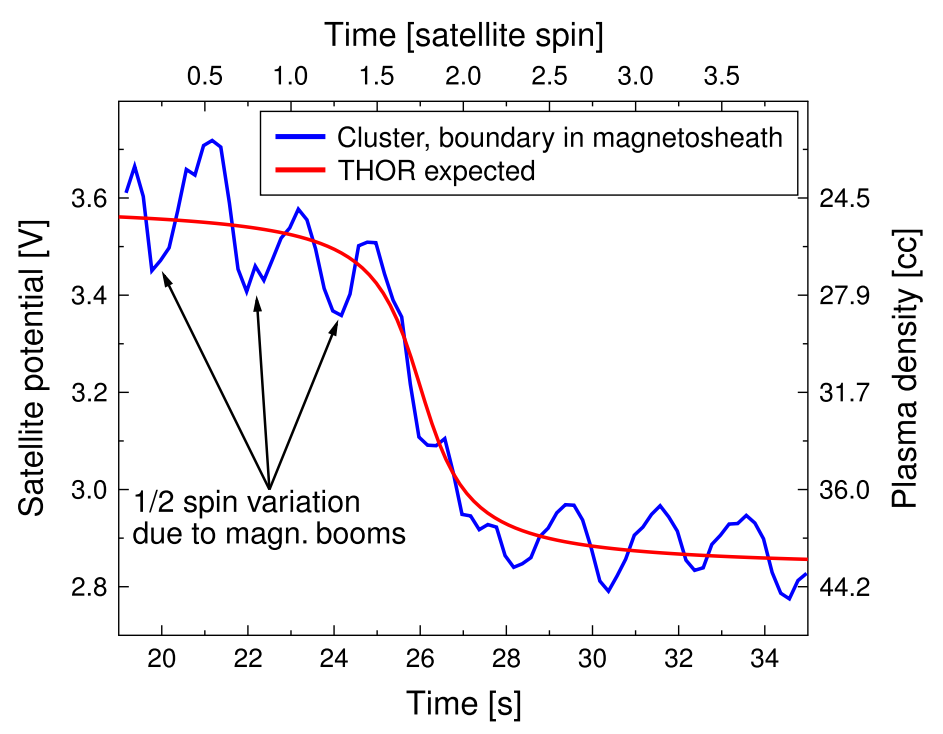

FIGURE 8. THOR expected satellite potential across a density gradient compared to Cluster data. The Sun-pointing orientation will significantly reduce disturbances in the satellite potential due to spin and thus allow quantitative assessment of the density gradients down to electron scales. This will also allow very accurate estimates of the turbulent density fluctuations in the kinetic range.

$\begin{array}{lccc} & 4 \times 16 R_{E} & 4 \times 26 R_{E} & 14 \times 60 R_{E} \\ & \text { Survey } & & \\ \text { Fraction of total telemetry (\%) } & 9 & 20 & 24 \\ \text { Rate (kbps) } & 29.9 & 29.9 & 6.0 \\ \text { Data (Gbit/orbit) } & 3.9 & 7.4 & 6.5 \\ & \text { Burst } & & \\ \text { Data rate (kbps) } & 4651 & 4299 & 4299 \\ \text { Downloaded (min) } & 139 & 113 & 76 \\ \text { Downloaded (Gbit/orbit) } & 36.9 & 27.8 & 18.6 \\ & & & \\ \text { Data rate (kbps) } & \text { HRWF } & & \\ \text { Downloaded (s) } & 465 & 430 & 430 \\ \text { Downloaded (Gbit/orbit) } & 146 & 110 & 74 \\ & 4.1 & 3.1 & 2.1 \\ \text { Downloaded (Gbit/orbit) } & \text { Total } & & \\ & 44.9 & 38.3 & 27.2\end{array}$

TABLE 5. THOR data rates, showing at which rates data are recorded in the spacecraft mass memory.

Table 5 gives a representative instrument telemetry budget appropriate for the primary science objectives. Survey data provide a continuous low telemetry stream of field and particle data, which can be downloaded quickly to ground and is intended primarily to be used as a basis for selection of Burst intervals. It can, however, be 


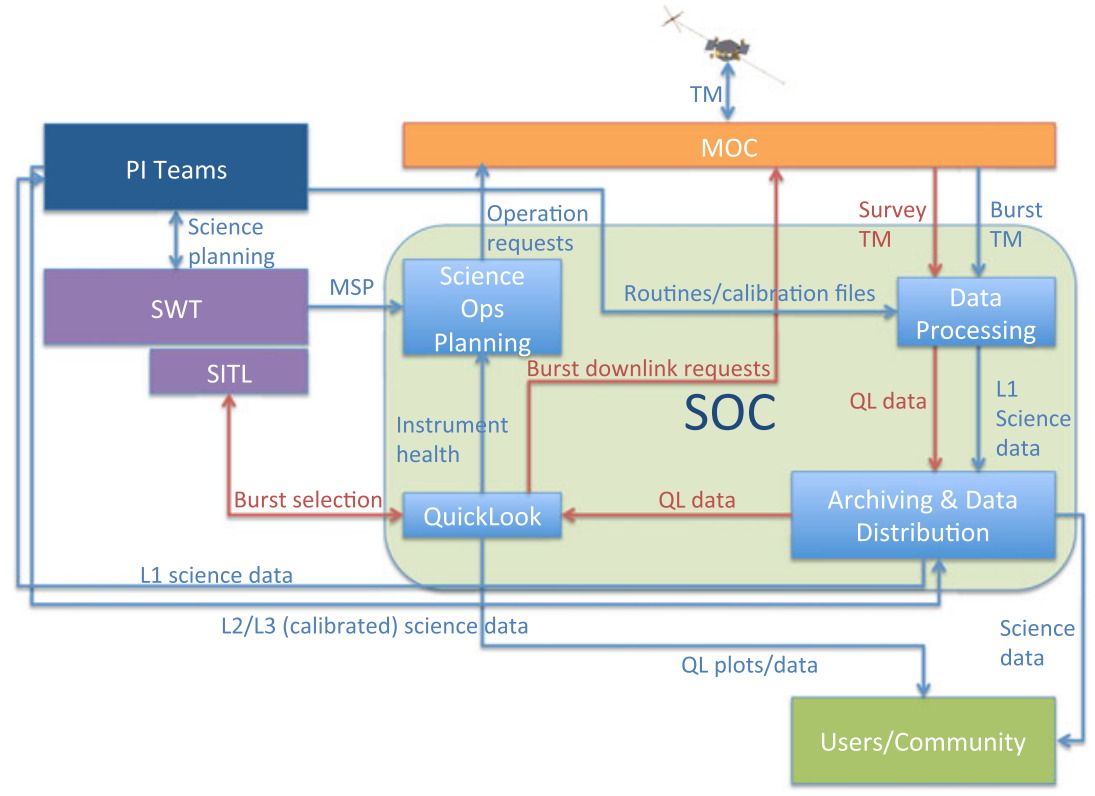

FIgURE 9. Possible THOR Science Ground Segment structure. Acronyms: Science Working Team (SWT), Scientist in the Loop (SITL), Mission Operation Center (MOC), Science Operation Center (SOC), Master Science Plan (MSP).

also used for other science and calibration purposes. Survey data contain MAG, SCM and EFI time series at 32 samples per second (sps), spectra of electric and magnetic fields, as well as three-dimensional distributions and moments for ions and electrons at up to 4 times per spin $(7.5 \mathrm{sps})$. Survey data constitute $10 \%$ to $20 \%$ of the total downlinked telemetry. The baseline is to change the payload modes by use of a single command from the spacecraft which triggers execution of predefined sequences (macros) stored in the flash memory of the PPU and FWP.

\section{Science operations}

Figure 9 illustrates a possible structure of the Science Ground Segment (SGS). The baseline for the THOR science operations is to collect and save on-board high-resolution Burst data for parts of the orbit corresponding to a ROI defined by the mission science requirements, see figure 10. A relatively small fraction of the Burst data can be transmitted to ground due to telemetry limitations. Selection of scientifically interesting Burst intervals for downlink is done on the ground based on the Survey stream, which is being saved on-board during the entire period when the spacecraft payload is operating. During the ground contact, all of the Survey data and, in addition, Burst data from the previously selected intervals are transmitted. Identification of the interesting time intervals from which to download Burst data are based on inspection of the Survey data as well as the ancillary data transmitted with the Survey stream. The Burst data are then split into intervals and a figure of merit (FM) is assigned to each of the intervals, so that the intervals with higher FM will have higher priority in the downlink queue. Selection of intervals can be done by an automatic algorithm at SOC, or by the Scientist In The Loop (SITL). The SITL 


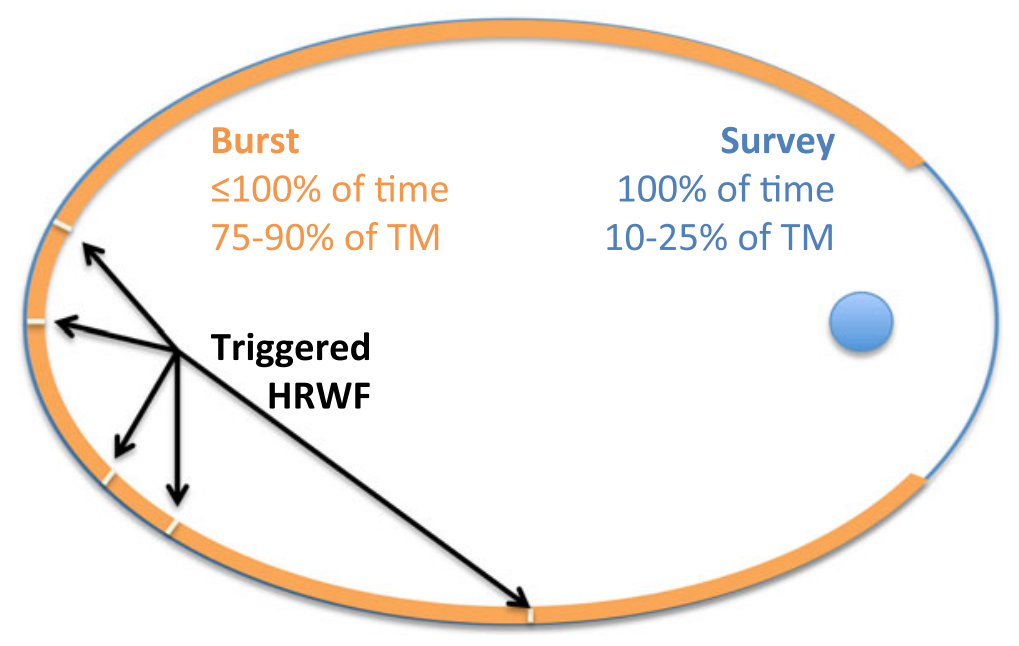

FIGURE 10. Burst and Survey mode acquisition along the orbit.

will be able to carry out his/her responsibilities remotely over the internet. A similar strategy involving a SITL is employed by the NASA MMS and Van Allen Probes missions. In case SITL selection is not available at the moment the SOC needs to generate the telecommands for Burst downlink, the intervals and FMs produced by the default automatic algorithm will be used. The amount of on-board storage allows 8-10 days of Burst data to be stored, which defines the time frame for the SOC/SITL to make decisions about Burst selection.

THOR will employ an Open Data policy starting from 6 months into the nominal operations (after the end of commissioning). This 6 month delay is needed for the instrument teams to establish the data processing and calibration pipelines. After the end of the active phase of the mission we would expect the long-term archive to be available at ESA.

\section{Current status and outlook}

Currently THOR is undergoing a study within ESA, also involving industry and the payload teams. As a result of the study, the specific implementation details will be refined and the final mission will differ in some aspects from the concept presented here. In parallel, there are large on-going efforts on the numerical simulation side to support the THOR study phase activities. The study phase will end in the beginning of 2017. In June 2017 one of the three candidate missions (THOR, ARIEL or XIPE) will be selected for the implementation phase.

The plasma turbulence community is a large cross-disciplinary science community. Carefully designed laboratory plasma experiments, as well as increasingly sophisticated numerical simulations, will complement the measurements we propose to conduct with THOR. The totality of those efforts will lead to a paradigm shift in our understanding of plasma turbulence and energization mechanisms in astrophysical plasmas.

\section{Acknowledgements}

The THOR science team thanks the Swedish National Space Board for support to carry out a technical assessment phase study before the proposal submission. 
We acknowledge the useful discussion and comments from the THOR team (http://thor.irfu.se/team) and particularly D. Delcourt, D. Fontaine, A. Kis, G. Lapenta, M. Maksimovic, M. Opher, G. Paschmann, A. Petrukovic, S. Schwartz. We acknowledge: the support of the UK Space Agency through grant ST/N003322/1 to ICL; the support of Agenzia Spaziale Italiana through contract ASI-INAF 2015-039-R.O to University of Calabria, Italy and at IAPS/INAF, Rome; the support of the Belgian Science Policy Office through PRODEX PEA 4000116805 to BIRA-IASB; the support of the Czech Science Foundation through project 16-04956S to Charles University, Prague; the support of ESA PRODEX to IAP Prague; the support of CNRS and CNES to IRAP, LPP, LP2CE and LESIA; the support of the German Space Agency through grant 50 OO 1603 to CAU; the support of Swedish National Space Board through grants $232 / 15$ and 257/15 to IRF, Uppsala; the support of Academy of Finland through grant 267144 and European Research Council Consolidator through grant 682068-PRESTISSIMO to FMI; the support of ISSI team 'Kinetic Turbulence and Heating in the Solar wind'; the support of FP7 projects STORM and SHOCK. Vlasiator (http://vlasiator.fmi.fi) has been developed with the support of Academy of Finland and European Research Council Starting grant 200141-QuESpace.

\section{REFERENCES}

von Alfthan, S., Pokhotelov, D., Kempf, Y., Hoilijoki, S., Honkonen, I., Sandroos, A. \& PALmRoth, M. 2014 Vlasiator: first global hybrid-Vlasov simulations of Earth's foreshock and magnetosheath. J. Atmos. Sol.-Terr. Phys. 120, 24-35.

BorovsKy, J. E. \& GARY, S. P. 2014 How important are the alpha-proton relative drift and the electron heat flux for the proton heating of the solar wind in the inner heliosphere? J. Geophys. Res. 119 (7), 2014JA019758.

Burgess, D., Lucek, E. A., Scholer, M., Bale, S. D., Balikhin, M. A., Balogh, A., Horbury, T. S., Krasnoselskikh, V. V., Kucharek, H., Lembège, B. et al. 2005 Quasi-parallel shock structure and processes. Space Sci. Rev. 118 (1-4), 205-222.

Camporeale, E. \& Burgess, D. 2011 The dissipation of solar wind turbulent fluctuations at electron scales. Astrophys. J. 730 (2), 114.

CAPrioli, D. \& SPITKOVSKY, A. 2014 Simulations of ion acceleration at non-relativistic shocks. I. Acceleration efficiency. Astrophys. J. 783 (2), 91.

Chandran, B. D. G., Li, B., Rogers, B. N., Quataert, E. \& Germaschewski, K. 2010 Perpendicular ion heating by low-frequency Alfvén-wave turbulence in the solar wind. Astrophys. J. 720 (1), 503.

Chen, C. H. K., Sorriso-Valvo, L., ŠAfránková, J. \& NěMeČeK, Z. 2014 Intermittency of solar wind density fluctuations from ion to electron scales. Astrophys. J. Lett. 789 (1), L8.

Eastwood, J. P., Lucek, E. A., Mazelle, C., Meziane, K., Narita, Y., Pickett, J. \& Treumann, R. A. 2005 The foreshock. Space Sci. Rev. 118 (1-4), 41-94.

Haynes, C. T., Burgess, D. \& CAmporeale, E. 2014 Reconnection and electron temperature anisotropy in sub-proton scale plasma turbulence. Astrophys. J. 783 (1), 38.

Karimabadi, H., Roytershteyn, V., Wan, M., Matthaeus, W. H., Daughton, W., Wu, P., Shay, M., Loring, B., Borovsky, J., Leonardis, E. et al. 2013 Coherent structures, intermittent turbulence, and dissipation in high-temperature plasmas. Phys. Plasmas 20 (1), 012303.

Kasper, J. C., Lazarus, A. J. \& Gary, S. P. 2008 Hot solar-wind helium: direct evidence for local heating by Alfvén-Cyclotron dissipation. Phys. Rev. Lett. 101 (26), 261103.

LiU, Y., Richardson, J. D., Belcher, J. W. \& KASPER, J. C. 2007 Temperature anisotropy in a shocked plasma: mirror-mode instabilities in the heliosheath. Astrophys. J. Lett. 659 (1), L65. 
Lucek, E. A., Constantinescu, D., Goldstein, M. L., Pickett, J., Pinçon, J. L., Sahraoui, F., Treumann, R. A. \& Walker, S. N. 2005 The magnetosheath. Space Sci. Rev. 118 (1-4), 95-152.

Lundin, R., Haerendel, G. \& Grahn, S. 1994 The Freja science mission. Space Sci. Rev. 70, 405-419.

MARSCH, E. 2006 Kinetic physics of the solar corona and solar wind. Living Rev. Solar Phys. 3, 1.

Perri, S., Goldstein, M. L., Dorelli, J. C. \& Sahraoui, F. 2012 Detection of small-scale structures in the dissipation regime of solar-wind turbulence. Phys. Rev. Lett. 109 (19), 191101.

Perrone, D., Valentini, F., Servidio, S., Dalena, S. \& Veltri, P. 2013 Vlasov simulations of multi-ion plasma turbulence in the solar wind. Astrophys. J. 762 (2), 99.

Perrone, D., Valentini, F., Servidio, S., Dalena, S. \& Veltri, P. 2014 Analysis of intermittent heating in a multi-component turbulent plasma. Eur. Phys. J. D 68, 209.

Retinò, A., Sundkvist, D., Vaivads, A., Mozer, F., André, M. \& Owen, C. J. 2007 In situ evidence of magnetic reconnection in turbulent plasma. Nat. Phys. 3, 236-238.

Richardson, J. D., Paularena, K. I., Lazarus, A. J. \& Belcher, J. W. 1995 Radial evolution of the solar wind from IMP 8 to Voyager 2. Geophys. Res. Lett. 22 (4), 325-328.

ŠAfránková, J., NĚmeČek, Z., Přech, L. \& Zastenker, G. N. 2013 Ion kinetic scale in the solar wind observed. Phys. Rev. Lett. 110 (2), 025004.

Sahraoui, F., Goldstein, M. L., Robert, P. \& Khotyaintsev, Yu. V. 2009 Evidence of a cascade and dissipation of solar-wind turbulence at the electron gyroscale. Phys. Rev. Lett. 102 (23), 231102.

Servidio, S., Matthaeus, W. H., Shay, M. A., Cassak, P. A. \& Dmitruk, P. 2009 Magnetic reconnection in two-dimensional magnetohydrodynamic turbulence. Phys. Rev. Lett. 102 (11), 115003.

Servidio, S., Valentini, F., Califano, F. \& Veltri, P. 2012 Local kinetic effects in twodimensional plasma turbulence. Phys. Rev. Lett. 108 (4), 045001.

Soucek, J., Ahlen, L., Bale, S., Bonnell, J., Boudin, N., Brienza, D., Carr, C., Cipriani, F., Escoubet, C. P., Fazakerley, A. et al. 2016 EMC aspects of turbulence heating observer (THOR) spacecraft. In 2016 ESA Workshop on Aerospace EMC (Aerospace $E M C)$, pp. 1-4.

Sundkvist, D., Retinò, A., Vaivads, A. \& Bale, S. D. 2007 Dissipation in turbulent plasma due to reconnection in thin current sheets. Phys. Rev. Lett. 99 (2), 025004.

Tenbarge, J. M. \& Howes, G. G. 2013 Current sheets and collisionless damping in kinetic plasma turbulence. Astrophys. J. Lett. 771 (2), L27.

Vaivads, A., Andersson, G., Bale, S. D., Cully, C. M., Keyser, J. De, Fujimoto, M., Grahn, S., HaAland, S., Ji, H., Khotyaintsev, Y. V. et al. 2011 EIDOSCOPE: particle acceleration at plasma boundaries. Exp. Astron. 33 (2-3), 491-527.

Valentini, F., Servidio, S., Perrone, D., Califano, F., Matthaeus, W. H. \& Veltri, P. 2014 Hybrid Vlasov-Maxwell simulations of two-dimensional turbulence in plasmas. Phys. Plasmas 21 (8), 082307. 